\title{
GJA1 Gene
}

National Cancer Institute

\section{Source}

National Cancer Institute. G/A1 Gene. NCI Thesaurus. Code C115170.

This gene is involved in gap junction assembly. 